\title{
ENERGY SCHEDULING IN A HYBRID NETWORK OF MULTI-MICROGRIDS
}

\author{
Khushboo Jain \\ Department of Computer Science and Engineering, \\ Indian Institute of Information Technology, \\ Nagpur, Maharashtra, India \\ khushboo.jain@iiitn.ac.in \\ http://iiitn.ac.in \\ Meera Dhabu \\ Department of Computer Science and Engineering, \\ Visvesvaraya National Institute of Technology, \\ Nagpur, Maharashtra, India \\ meeradhabu@cse.vnit.ac.in \\ https://vnit.ac.in/ \\ Omprakash Kakde \\ Director, \\ Department of Computer Science and Engineering, \\ Indian Institute of Information Technology, \\ Nagpur, Maharashtra, India \\ director@iiitn.ac.in \\ http://iiitn.ac.in
}

\section{Abstract}

One of the most significant responsibilities in a multi-microgrid system is optimizing energy scheduling since it is an effective technique to improve technical and financial performance. It is extremely difficult to effectively schedule energy due to the inclusion of a wide range of dispersed load and generation in various microgrids. This paper presents energy scheduling strategy in a hybrid multimicrogrid system, which covers different components of a society in a growing city, such as community, single-dwelling, industry, and campus unit, all of which have different types of generation units. For energy scheduling between different microgrid systems, the significance of energy market prices is addressed in a distinct way. The simulation results and discussions demonstrate that suggested strategy reduces cost of buying energy from neighboring microgrids compared to when individual microgrid purchase energy from the utility grid.

Keywords: Optimization, Energy Scheduling, Multi-microgrid, Energy Market, Distributed Generation

$\begin{array}{cl}\text { Nomenclature } & \\ \text { MG } & \text { Microgrid } \\ \text { PV } & \text { Photovoltaics } \\ \text { CHP } & \text { Combined Heat and Power } \\ \text { ESS } & \text { Energy Storage System } \\ \text { EV } & \text { Electric Vehicle } \\ \mathrm{t} & \text { Time slot }(\mathrm{t} \in\{1,2,3, \ldots, 24\}) \\ E_{C H P_{-} I N D}^{i, t} & \text { Amount of Energy Produced by CHP of } \mathrm{i}^{\text {th }} \text { Industry microgrid at time slot } \mathrm{t} \\ E_{P V_{-} I N D}^{i, t} & \text { Amount of Energy Produced by PV of } \mathrm{i}^{\text {th }} \text { Industry microgrid at time slot } \mathrm{t}\end{array}$




\begin{tabular}{|c|c|}
\hline$E_{E S S_{-} C O M}^{i, t}$ & Amount of Energy Present in ESS of $i^{\text {th }}$ Community microgrid at time slot $t$ \\
\hline$E_{P V_{-} C O M}^{i, t}$ & Amount of Energy Produced by PV of $i^{\text {th }}$ Community microgrid at time slot $t$ \\
\hline$E_{E S S_{-} C A M P}^{i, t}$ & Amount of Energy Present in ESS of $\mathrm{i}^{\text {th }}$ Campus microgrid at time slot $\mathrm{t}$ \\
\hline$E_{C H P \_C A M P}^{i, t}$ & Amount of Energy Produced by CHP of $i^{\text {th }}$ Campus microgrid at time slot $t$ \\
\hline$E_{P V_{-} C A M P}^{i, t}$ & Amount of Energy Produced by PV of $\mathrm{i}^{\text {th }}$ Campus microgrid at time slot $\mathrm{t}$ \\
\hline$E_{P V \_S D}^{i, t}$ & Amount of Energy Produced by PV of $i^{\text {th }}$ Single-Dwelling microgrid at time slot $t$ \\
\hline$E_{E V \_S D}^{i, t}$ & Amount of Energy Present in ESS of $i^{\text {th }}$ Single-Dwelling microgrid at time slot $t$ \\
\hline$L_{S D}^{i, t}$ & Load at $\mathrm{i}^{\text {th }}$ Single-Dwelling microgrid at time slot $\mathrm{t}$ \\
\hline$L_{I N D}^{i, t}$ & Load at $\mathrm{i}^{\text {th }}$ Industry microgrid at time slot $\mathrm{t}$ \\
\hline$L_{C O M}^{i, t}$ & Load at $\mathrm{i}^{\text {th }}$ Community microgrid at time slot $\mathrm{t}$ \\
\hline$L_{C A M P}^{i, t}$ & Load at $\mathrm{i}^{\text {th }}$ Campus microgrid at time slot $\mathrm{t}$ \\
\hline EV avail & Binary variable indicating if $\mathrm{EV}$ is available. \\
\hline$\rho_{G B P}^{t}$ & Utility Grid Buying Price at time slot $\mathrm{t}$ \\
\hline$\rho_{M B P}^{t}$ & Microgrid Grid Buying Price at time slot $\mathrm{t}$ \\
\hline$\rho_{M S P}^{t}$ & Microgrid Grid Selling Price at time slot t \\
\hline$\rho_{G S P}^{t}$ & Utility Grid Selling Price at time slot $\mathrm{t}$ \\
\hline$E_{I N D}^{t}$ & Excess Energy at Industry Microgrid \\
\hline$E_{\text {req_IND }}^{t}$ & Energy Requirement at Industry Microgrid \\
\hline$E_{C O M}^{t}$ & Excess Energy at Community Microgrid \\
\hline$E_{\text {req_COM }}^{t}$ & Energy Requirement at Community Microgrid \\
\hline$E_{C A M P}^{t}$ & Excess Energy at Campus Microgrid \\
\hline$E_{\text {req_CAMP }}^{t}$ & Energy Requirement at Campus Microgrid \\
\hline$E_{S D}^{t}$ & Excess Energy at Single-Dwelling Microgrid \\
\hline$E_{\text {req_SD }}^{t}$ & Energy Requirement at Single-Dwelling Microgrid \\
\hline$\rho_{C H P}^{t}$ & CHP Generation Cost per kWh \\
\hline$\eta^{c}$ & Charging Efficiency of ESS \\
\hline$\eta^{\mathrm{d}}$ & Discharging Efficiency of ESS \\
\hline$k^{t}$ & Binary variable indicating if ESS is charging/discharging \\
\hline$E_{E S S C O M_{-} D I S}^{i, t}$ & Discharging energy of ESS at Community Microgrid \\
\hline$E_{E S S C O M \_C H}^{i, t}$ & Charging energy of ESS at Community Microgrid \\
\hline$E_{E V_{-} D I S}^{i, t}$ & Discharging energy of EV at Community Microgrid \\
\hline
\end{tabular}




$\begin{array}{cl}E_{E V_{-} C H}^{i, t} & \text { Charging energy of EV at Community Microgrid } \\ E_{E S S C A M P_{-} C H}^{i, t} & \text { Charging energy of ESS at Campus Microgrid } \\ E_{E S S C A M P_{-} D I S}^{i, t} & \text { Discharging energy of ESS at Campus Microgrid } \\ E_{E S S M A X_{-} D I S}^{i, t} & \text { Maximum discharging energy of ESS } \\ E_{E S S M A X_{-} C H}^{i, t} & \text { Maximum charging energy of ESS } \\ E_{E S S M A X}^{i, t} & \text { Maximum Capacity of ESS } \\ E_{E S S M I N}^{i, t} & \text { Minimum Capacity of ESS } \\ E_{C H P M I N}^{i, t} & \text { Minimum CHP Generation } \\ E_{C H P M A X}^{i, t} & \text { Maximum CHP Generation } \\ P_{\text {ch }}^{t} & \text { Willingness of ESS to charge at time } \mathrm{t} \\ P_{\text {dis }}^{t} & \text { Willingness of ESS to discharge at time t }\end{array}$

\section{Introduction}

Due to the increasing energy demand and the environment's growing greenhouse gas emissions, smart grid has arisen as one of the promising technologies. Distributed generation is an emerging concept that enables onsite production of electrical power. This system utilizes various energy sources such as solar panels and wind turbines. The concept of distributed generation systems (DG) has gained widespread acceptance due to its ability to integrate various types of energy sources. The AI motivated electrical grid will allow consumers to control their appliances remotely. This concept is expected to reduce energy consumption and increase transparency.

The concept of a microgrid is that it combines various distributed energy resources such as electric vehicles and energy storage systems to provide a uniform supply of energy to a user. When a MG's energy requirement exceeds its own consumption, it may offer the excess power to the utility grid or to neighboring MGs. This phenomenon triggers the need for energy trading among various microgrids. To inspire internal energy trading, many of the researchers have assumed that,

$$
\rho_{G B P}^{t}>\rho_{M B P}^{t} \geq \rho_{M S P}^{t}>\rho_{G S P}^{t}
$$

This supports trade of energy among microgrids. Hence, every MG that require energy will attempt to get their necessities fulfilled from the other nearby MGs that have overabundance energy instead of purchasing energy from the utility grid at more exorbitant cost. Be that as it may, microgrid created energy is restricted and discontinuous in nature. Accordingly, there is a need to effectively schedule microgrid generated energy among MGs.

Some researchers have carried a survey on different parameters of microgrid[1]-[6]. A. Hirsch et al. described the characteristics of MG along with an overview of MG energy generation and storage options[1]. F. Martin-Martínez et al. discussed the various physical devices used in MGs, as well as provides a study of the communication protocols used in MGs and an in-depth look at real-life MG experiences throughout the world[2]. A. H. Fathima et al. conducted a review on the state-of-the-art deployment of optimization tools and methodologies to microgrids that integrate renewable energy sources[3]. M. F. Zia et al. provide a comprehensive and critical examination of decision-making techniques and their solution methodologies for microgrid energy management systems. A cost-effective deployment of microgrid energy management systems is also explored, as a comparative study of communication technologies[4].

Many researches have used various auctioning techniques in the energy market. The use of a hybrid optimization method for distributed energy resource (DER) management in functioning of Smart Grid is presented by Ramachandran B et al. The method stresses the benefits of employing multiagent systems to run a Smart Grid in the energy market profitably[7]. Several scholars have developed game theoretic approach to help the MGs in the energy market to maximize their utility[8], [9]. Wang et al. suggested an energy management paradigm for a smart grid power system that decreases losses and maintains stability. It employs a loss reduction approach based on game theory to control distributed devices via a price[10]. W. Saad developed a noncooperative game between storage devices, such as electric vehicles or a battery array, that trade their stored energy[11]. The Stackelberg game has also been employed by some researchers for energy management. Liu et al. presented an hour-ahead 
pricing system for photovoltaic prosumers to share energy[12]. Lee et al. suggested an energy scheduling technique in which the market is considered to be fully distributed without the use of a central controller[13].

In some of the energy scheduling methods that have been studied so far, energy is scheduled to MGs that have a specific priority. Park et al. presented a contribution-based energy trading mechanism in a competitive market, based on previous levels of commitment, to encourage MGs to provide more and more energy in a microgrid system[14]. Jadhav et al. have presented a method for generating the priority index in which two factors are considered when allocating the priority to MG: the microgrid's verifiable pledges and the MG's historical demand[15]. Both Park et al. and Jadhav et al. suggest work that is well on its way to causing energy depletion and misfortune for some of the newer MGs. Funde et al. developed a starvation-free optimal microgrid generated energy scheduling policy for several of the latest MGs (SF-OEAP). Funde et al. proposed three new parameters: net load forecast prediction accuracy, microgrid revenue index, and hunger parameter. Although the problem of starvation has been eliminated by introducing a starvation parameter to SF-OEAP energy scheduling, the energy distribution is still unequal because the priority is computed using several parameters[16].

Despite there are some promising researches on the multi-microgrid system, there remain a few significant research gaps. Regardless of the fact that microgrid energy scheduling has been done for diverse purposes using various approaches in recent works, researchers have only considered a specific type of multi-microgrid system. In [17], authors have only considered energy storage systems. Whereas in the proposed work, a hybrid multimicrogrid system consisting of different types microgrid is taken into account. Here, hybrid microgrid refers to the microgrid having more than one type of renewable energy generator. This is because, within the society in growing city there can be microgrids having different types of renewable generator including PV, CHP, etc. and energy storage systems. Therefore, four common types of microgrids that are considered in this work are: community microgrid, industry microgrid, single-dwelling microgrid and campus microgrid. The energy that is generated through CHP is also controlled depending upon the CHP energy generation cost and energy trading prices. Finally, the energy is scheduled using an energy scheduling mechanism which schedules energy fairly amongst all microgrids.

The following are the contributions of this paper:

(1) The cost-utility of an MG may not be explained in the absence of optimization techniques. The goal of optimization is to find the optimum solution from a set of defined solutions that is the most economical or has the highest achievable efficiency under the constraints. Therefore, in this work we have applied optimization to each microgrid before scheduling the energy to reduce the cost of energy.

(2) Since we have considered different types of microgrid with different RES and storage, each microgrid has a different objective function for optimization keeping into account the renewable energy generators and the storage systems available with that microgrid.

(3) We have also considered the CHP energy generation cost and willingness of the ESS/EV to charge/discharge depending upon the cost of energy during energy scheduling.

(4) The energy scheduling mechanism that is use to schedule energy amongst these microgrids reduces the cost of buying energy for the buyer MGs and also the seller MGs are profited by fair distribution of revenue. 


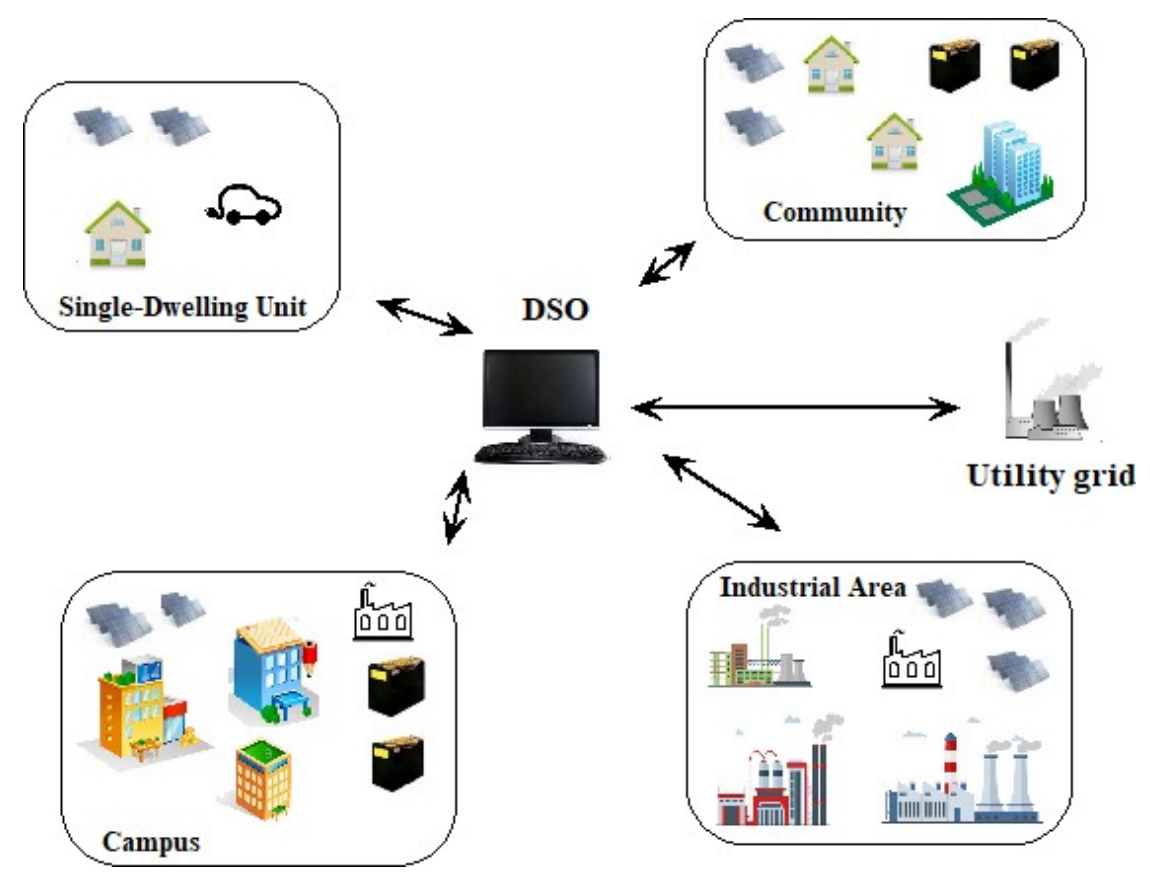

Fig. 1. System Model

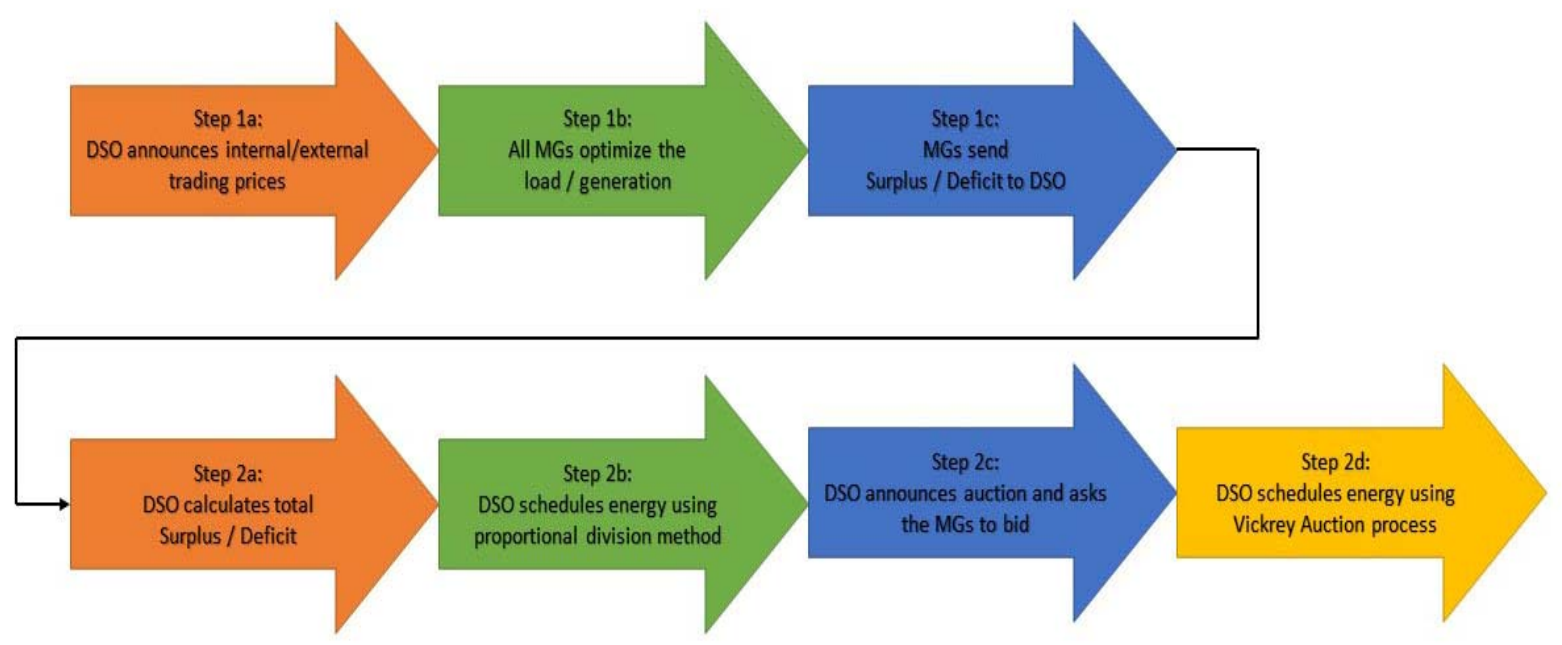

Fig. 2. Step Diagram of the proposed strategy

\section{System Model}

Fig. 1. shows the system model that is considered in this work for energy scheduling. There are four types of microgrids in the system model: community microgrid, industry microgrid, campus microgrid and singledwelling unit microgrid. These microgrids have PV and/or CHP as renewable energy generators and ESS or EV as energy storage. Each MGs is interconnected and can trade energy with other microgrid and with utility grid through energy trading lines. All the essential communication setup is assumed to be available in the network of MGs.

In community microgrid there are various residential units like single house and buildings. This microgrid has PV for energy generation and ESS for energy storage. In campus microgrids there are various campus buildings and residential buildings for faculties and students. The campus microgrid has PV and CHP for energy generation and ESS for energy storage. The third type of microgrid is single-dwelling unit which consists of a single resident that has EV. Lastly, the industry microgrid consists of various industries and has CHP for energy generation and ESS for energy storage.

Depending upon the time of the day, the energy requirement of all the microgrids can vary and therefore a microgrid can have shortage of energy or excess energy that needs to be properly scheduled among neighboring microgrids. All the microgrids are connected to a DSO which is responsible for energy scheduling among the microgrids. In this paper, it is assumed that the predicted output of PV is reasonably accurate so we center around the control of CHP. The proposed mechanism works in two steps. 
In step 1 , DSO announces $\rho_{G S P}^{t}, \rho_{M S P}^{t}, \rho_{M B P}^{t}$ and $\rho_{G B P}^{t}$ to the microgrids. Each microgrid performs optimization and decides the excess or shortfall energy depending on CHP energy production cost $\left(\rho_{C H P}^{t}\right)$, internal energy trading cost $\left(\rho_{M S P}^{t} / \rho_{M B P}^{t}\right)$ and the local load requirement of $\mathrm{MG}\left(L_{S D}^{i, t} / L_{I N D}^{i, t} / L_{C A M P}^{i, t} / L_{C O M}^{i, t}\right)$. This excess or shortfall energy is sent to the DSO. In step 2, DSO schedules energy fairly amongst all buyer microgrid. Fig. 2. shows the details of the steps that are performed in the proposed strategy.

\section{Proposed Mechanism}

In the proposed work different types of microgrids viz. campus microgrid, community microgrid, singledwelling microgrid and industry microgrid are assumed and only the electric energy of CHP is considered. The $\mathrm{ESS} / \mathrm{EV}$, if present, can buy or sell energy depending upon the internal energy trading prices $\left(\rho_{M S P}^{t}\right.$ and $\left.\rho_{M B P}^{t}\right)$.

\subsection{Step 1: Optimization}

If the energy generation of a microgrid is greater than the energy requirement, then the microgrid acts as a seller and if the generation is less than the energy requirement, then the microgrid acts as a buyer. Each buyer microgrid tries to minimize the energy buying cost and each seller microgrid tries to maximize the profit of selling energy through internal/external trading.

\begin{tabular}{|l|l|l|}
\hline $\begin{array}{l}\text { Sr. } \\
\text { No. }\end{array}$ & Microgrid Type & Microgrid \\
\hline 1 & Industry MG & $\mathrm{MG}_{1}, \mathrm{MG}_{2}$ \\
\hline 2 & Community MG & $\mathrm{MG}_{3}, \mathrm{MG}_{4}, \mathrm{MG}_{5}$ \\
\hline 3 & Single-Dwelling MG & $\mathrm{MG}_{6}, \mathrm{MG}_{7}, \mathrm{MG}_{8}, \mathrm{MG}_{9}$ \\
\hline 4 & Campus MG & $\mathrm{MG}_{10}$ \\
\hline
\end{tabular}

Table 1 Microgrids in the System

\begin{tabular}{|c|c|c|c|c|c|c|c|c|c|c|}
\hline $\begin{array}{l}\text { Time } \\
\text { Slot }\end{array}$ & $\mathbf{M G}_{1}$ & $\mathbf{M G}_{2}$ & $\mathbf{M G}_{3}$ & $\mathbf{M G}_{4}$ & $\mathbf{M G}_{5}$ & MG6 $_{6}$ & $\mathbf{M G}_{7}$ & MG8 $_{8}$ & MG9 & $\mathbf{M G}_{10}$ \\
\hline 1 & 11 & 65 & -25 & -11 & -64 & -3 & -2 & -4 & -1 & 54 \\
\hline 2 & 15 & 65 & -20 & -12 & -64 & -7 & -2 & -3 & -1 & 57 \\
\hline 3 & 13 & 65 & -22 & -14 & -68 & -4 & -2 & -3 & -2 & 52 \\
\hline 4 & 10 & 65 & -26 & -11 & -70 & -2 & -3 & -2 & -1 & 50 \\
\hline 5 & 12 & 65 & -19 & -13 & -74 & -2 & -1 & -5 & -3 & 51 \\
\hline 6 & 28 & 65 & -28 & -10 & -85 & -3 & -1 & -4 & -2 & 46 \\
\hline 7 & 30 & 82 & -34 & -27 & -84 & -7 & -3 & -2 & -2 & 39 \\
\hline 8 & 36 & 91 & -12 & -30 & -102 & -5 & -2 & -1 & -4 & 35 \\
\hline 9 & 62 & 0 & -11 & -30 & -85 & 9 & 4 & 9 & 5 & 0 \\
\hline 10 & 86 & 0 & 0 & -34 & -54 & 5 & 2 & 11 & 3 & 0 \\
\hline 11 & 114 & 0 & 0 & -28 & -51 & 4 & 6 & 13 & 4 & 0 \\
\hline 12 & 130 & 0 & 0 & -22 & -62 & 2 & 5 & 12 & 6 & 0 \\
\hline 13 & 130 & 0 & 0 & -31 & -68 & 2 & 3 & 15 & 5 & 10 \\
\hline 14 & 72 & 0 & 0 & -38 & -53 & 3 & 1 & 17 & 3 & 5 \\
\hline 15 & 59 & 0 & 0 & -36 & -62 & 1 & 0 & 15 & 2 & 11 \\
\hline 16 & 43 & 0 & -8 & -21 & -68 & 3 & 1 & 12 & 2 & 23 \\
\hline 17 & 26 & 0 & -13 & -23 & -48 & 0 & 0 & 13 & 1 & 27 \\
\hline 18 & 27 & 0 & -11 & -24 & -42 & 0 & 1 & 8 & 1 & 21 \\
\hline 19 & 21 & 0 & $\begin{array}{l}-19 \\
\end{array}$ & -18 & -52 & -6 & -3 & -1 & -2 & 19 \\
\hline 20 & 22 & 90 & -24 & -15 & -69 & -8 & -2 & -1 & -3 & 20 \\
\hline 21 & 17 & 72 & -22 & -15 & -57 & -7 & -1 & -3 & -1 & 17 \\
\hline 22 & 14 & 66 & -20 & -17 & -42 & -9 & -2 & -3 & -4 & 14 \\
\hline 23 & 10 & 61 & -28 & -14 & -49 & -5 & -2 & -4 & -3 & 15 \\
\hline 24 & 10 & 63 & -30 & -10 & -52 & -6 & -2 & -2 & -3 & 19 \\
\hline
\end{tabular}




\subsubsection{Community Microgrid}

Community microgrid consist of PV for energy generation and ESS for energy storage. When the load is less than generation, the energy generated by PV can be used to charge the ESS. When the ESS is fully charged then the surplus energy of PV can be sold in the energy market. This is because it is non-dispatchable and can be utilized on demand. Excess energy at community MG can be calculated as,

$$
E_{\text {COM }}^{t}=E_{P V_{-} C O M}^{i, t}-L_{C O M}^{i, t}-E_{E S S C O M_{-} C H}^{i, t}
$$

When the load is greater than the energy generated by PV, the load demand can be satisfied by discharging of ESS depending upon its current status and energy trading prices. The willingness of the ESS to charge / discharge can be calculated as [18],

$$
\begin{array}{lr}
P_{c h}^{t}=\frac{\max \left(\rho^{M B P}\right)_{T}-\rho_{M B P}^{t}}{\max \left(\rho^{M B P}\right)_{T}-\min \left(\rho^{M B P}\right)_{T}} & \mathrm{~T}=1,2,3, \ldots, 24 \\
P_{d i s}^{t}=\frac{\rho_{M B P}^{t}-\min \left(\rho^{M B P}\right)_{T}}{\max \left(\rho^{M B P}\right)_{T}-\min \left(\rho^{M B P}\right)_{T}} & \mathrm{~T}=1,2,3, \ldots, 24
\end{array}
$$

The energy requirement at the community MG can be calculated as,

$$
\begin{aligned}
& E_{\text {req_COM }}^{t}=L_{\text {COM }}^{i, t}-E_{P V_{-} C O M}^{i, t}-\alpha \cdot E_{E S S C O M_{-} D I S}^{i, t} \\
& \alpha=\left\{\begin{array}{l}
0, P_{d i s}^{t}<0.5 \\
1, P_{\text {dis }}^{t} \geq 0.5
\end{array}\right.
\end{aligned}
$$
be,

To minimize the energy cost is the main goal of the community microgrid. Thus, the objective function will

$\operatorname{Min} \sum_{t}\left(\rho_{t}^{M B P} \cdot E_{\text {req_COM }}^{t}-\rho_{t}^{M S P} \cdot E_{\text {COM }}^{t}\right)$

Subject to,

$$
\begin{aligned}
& E_{P V_{-} C O M}^{i, t}+E_{E S S C O M_{-} D I S}^{i, t}+E_{\text {req_COM }}^{t}=L_{\text {COMP }}^{i, t}+E_{E S S C O M_{-} C H}^{i, t}+E_{C O M}^{t} \\
& 0 \leq E_{E S S C O M_{-} C H}^{i, t} \leq k^{t} \cdot E_{E S S M A X_{-} C H}^{i, t} \\
& 0 \leq E_{E S S C O M_{-} D I S}^{i, t} \leq\left(1-k^{t}\right) \cdot E_{E S S M A X_{-} D I S}^{i, t} \\
& k^{t}=\left\{\begin{array}{l}
1, \text { if ESS is charged } \\
0, \text { if ESS is discharged }
\end{array}\right. \\
& E_{E S S_{-} C O M}^{i, t}=E_{E S S_{-} C O M}^{i, t-1}+\eta^{c} \cdot E_{E S S C O M_{-} C H}^{i, t}-\eta^{d} \cdot E_{E S S C O M_{-} D I S}^{i, t} \\
& E_{\text {ESSMIN }}^{i, t} \leq E_{\text {ESS_COM }}^{i, t} \leq E_{\text {ESSMAX }}^{i, t}
\end{aligned}
$$
to (11).

Eq. (6) indicates load balancing constraint. The ESS framework applied in this work is described by Eq. (7)

\subsubsection{Industry Microgrid}

Industry microgrid consist of PV and CHP for energy generation. We assume that ESS is not present in this type of microgrid. When the load is less than generation, the PV generator can sell surplus energy to the grid. This is because it is non-dispatchable and can be utilized on demand. Excess energy at industry MG can be calculated as,

$$
E_{I N D}^{t}=E_{C H P_{I} N D}^{i, t}+E_{P V_{I} N D}^{i, t}-L_{I N D}^{i, t}
$$


When the load is greater than the energy generation, then the energy requirement can be calculated as,

$$
E_{\text {req_IND }}^{t}=L_{I N D}^{i, t}-E_{C H P_{-} I N D}^{i, t}-E_{P V \_I N D}^{i, t}
$$

The objective here is to minimize the energy cost,

$$
\operatorname{Min} \sum_{t}\left(\rho_{C H P}^{t} \cdot E_{C H P_{I} I N D}^{i, t}\right)+\sum_{t}\left(\rho_{M B P}^{t} \cdot E_{r e q_{-} I N D}^{t}-\rho_{M S P}^{t} \cdot E_{I N D}^{t}\right)
$$

Subject to,

$$
\begin{aligned}
& E_{C H P_{-} I N D}^{i, t}+E_{P V_{-} I N D}^{i, t}+E_{\text {req_IND }}^{t}=L_{I N D}^{i, t}+E_{I N D}^{t} \\
& E_{C H P M I N}^{i, t} \leq E_{\text {CHP_IND }}^{i, t} \leq E_{\text {CHPMAX }}^{i, t}
\end{aligned}
$$

CHP generation depends on internal energy trading prices $\left(\rho_{M S P}^{t}\right.$ and $\left.\rho_{M B P}^{t}\right)$. Therefore, there are three cases: 4. 1.CHP generation cost $\left(\rho_{C H P}^{t}\right)$ is greater than internal energy trading prices $\left(\rho_{M S P}^{t}\right.$ and $\left.\rho_{M B P}^{t}\right)$

When $\rho_{C H P}^{t}$ is highest compared to $\left(\rho_{M S P}^{t}\right.$ and $\left.\rho_{M B P}^{t}\right)$, CHP should generate energy to least of its capacity. If this CHP generated energy is greater than the load, then there will be excess energy that can be sold during internal energy trading.

\section{2. CHP generation cost $\left(\rho_{C H P}^{t}\right)$ is between microgrid energy buying price $\left(\rho_{M B P}^{t}\right)$ and microgrid energy} selling price $\left(\rho_{M S P}^{t}\right)$

When $\rho_{C H P}^{t}$ is greater than $\rho_{M S P}^{t}$ and less than $\rho_{M B P}^{t}$, then CHP should generate energy equal to the load requirement.

\section{3.CHP generation cost $\left(\rho_{C H P}^{t}\right)$ is less than internal energy trading prices $\left(\rho_{M S P}^{t}\right.$ and $\left.\rho_{M B P}^{t}\right)$}

When $\rho_{C H P}^{t}$ is lowest compared to $\left(\rho_{M S P}^{t}\right.$ and $\left.\rho_{M B P}^{t}\right)$, CHP should generate energy to the maximum of its capacity. If this CHP generated energy is greater than the load, then there will be excess energy that can be sold during internal energy trading.

\subsubsection{Single-Dwelling Microgrid}

Single dwelling unit consists of PV for energy generation. It also has a EV which can store energy, but $\mathrm{EV}$ is not always available at the microgrid. Therefore, for charging and discharging we consider the time slot when $\mathrm{EV}$ is available which is indicated by $E V_{\text {avail }}$. The objective function for this microgrid is,

$$
\operatorname{Min} \sum_{t}\left(\rho_{M B P}^{t} \cdot E_{r e q \_S D}^{t}-\rho_{M S P}^{t} \cdot E_{S D}^{t}\right)
$$

Subject to,

$$
\begin{aligned}
& E_{P V_{-} S D}^{i, t}+E_{E V_{-} D I S}^{i, t}+E_{\text {req_SD }}^{t}=L_{S D}^{i, t}+E_{E V_{-} C H}^{i, t}+E_{S D}^{t} \\
& 0 \leq E_{E V_{-} C H}^{i, t} \leq k^{t} \cdot E_{E V M A X_{-} C H}^{i, t} \\
& 0 \leq E_{E V_{-} D I S}^{i, t} \leq\left(1-k^{t}\right) \cdot E_{E V M A X_{-} D I S}^{i, t} \\
& k^{t}=\left\{\begin{array}{l}
1, \text { if } E V \text { is charged } \\
0, \text { if } E V \text { is discharged }
\end{array}\right. \\
& E_{E V_{-} S D}^{i, t}=E_{E V_{-} S D}^{i, t-1}+\eta^{c} \cdot E_{E V_{-} C H}^{i, t}-\eta^{d} \cdot E_{E V_{-} D I S}^{i, t} \\
& E_{E V M I N}^{i, t} \leq E_{E V_{-} S D}^{i, t} \leq E_{E V M A X}^{i, t}
\end{aligned}
$$

The EV framework applied in this work is described by Eq. (19) to (22) 


\subsubsection{Campus Microgrid}

Campus microgrid consist of PV and CHP for energy generation and ESS for energy storage. The PV and CHP in campus microgrid are assumed to operate in the same manner as in industry microgrid. ESS framework is also same as in other microgrids.

The objective of campus microgrid is also to minimize energy cost,

$$
\begin{aligned}
& \text { Min } \sum_{t}\left(\rho_{C H P}^{t} \cdot E_{C H P_{-} C A M P}^{i, t}\right)+\sum_{t}\left(\rho_{M B P}^{t} \cdot E_{\text {req_CAMP }}^{t}-\rho_{M S P}^{t} \cdot E_{C A M P}^{t}\right) \\
& E_{P V_{-} C A M P}^{i, t}+E_{E S S C A M P_{-} D I S}^{i, t}+E_{C H P_{-} C A M P}^{i, t}+E_{\text {req_CAMP }}^{t}=L_{C A M P}^{i, t}+E_{E S S C A M P_{-} C H}^{i, t}+E_{C A M P}^{t} \\
& E_{C H P M I N}^{i, t} \leq E_{C H P_{-} C A M P}^{i, t} \leq E_{C H P M A X}^{i, t} \\
& 0 \leq E_{E S S C A M P_{-} C H}^{i, t} \leq k^{t} \cdot E_{E S S M A X_{-} C H}^{i, t} \\
& 0 \leq E_{E S S C A M P_{-} D I S}^{i, t} \leq\left(1-k^{t}\right) \cdot E_{E S S M A X_{-} D I S}^{i, t} \\
& k^{t}=\left\{\begin{array}{l}
1, \text { if ESS is charged } \\
0, \text { if ESS is diSCharged }
\end{array}\right. \\
& E_{E S S_{-} C A M P}^{i, t}=E_{E S S_{-} C A M P}^{i, t-1}+\eta^{c} \cdot E_{E S S C A M P_{-} C H}^{i, t}-\eta^{d} \cdot E_{E S S C A M P \_D I S}^{i, t} \\
& E_{E S S M I N}^{i, t} \leq E_{E S S_{-} C A M P}^{i, t} \leq E_{E S S M A X}^{i, t}
\end{aligned}
$$

\subsection{Step 2: Energy Scheduling}

The buyer and the seller MGs send the amount of energy required and the amount excess energy to the DSO, respectively. The DSO uses completely fair energy scheduling algorithm to schedule energy among the MGs in the network. The CFES algorithm works as follows[19]:

(1) DSO computes total excess energy to be scheduled.

(2) The total energy is divided into $\mathrm{N}$ equal parts ( $\mathrm{N}$ is the total number buyers in the network) by the DSO and is scheduled to the buyers.

(3) If the energy requirement of some buyers is not satisfied by this energy distribution, then the buyer bids for energy in auction process which is the second phase in CFES algorithm.

(4) DSO schedules the remaining energy, if any, according to the Vickrey auction process.

(5) If the energy requirement of few buyers is not satisfied even by auctioning, then the buyer purchases energy from the utility grid.

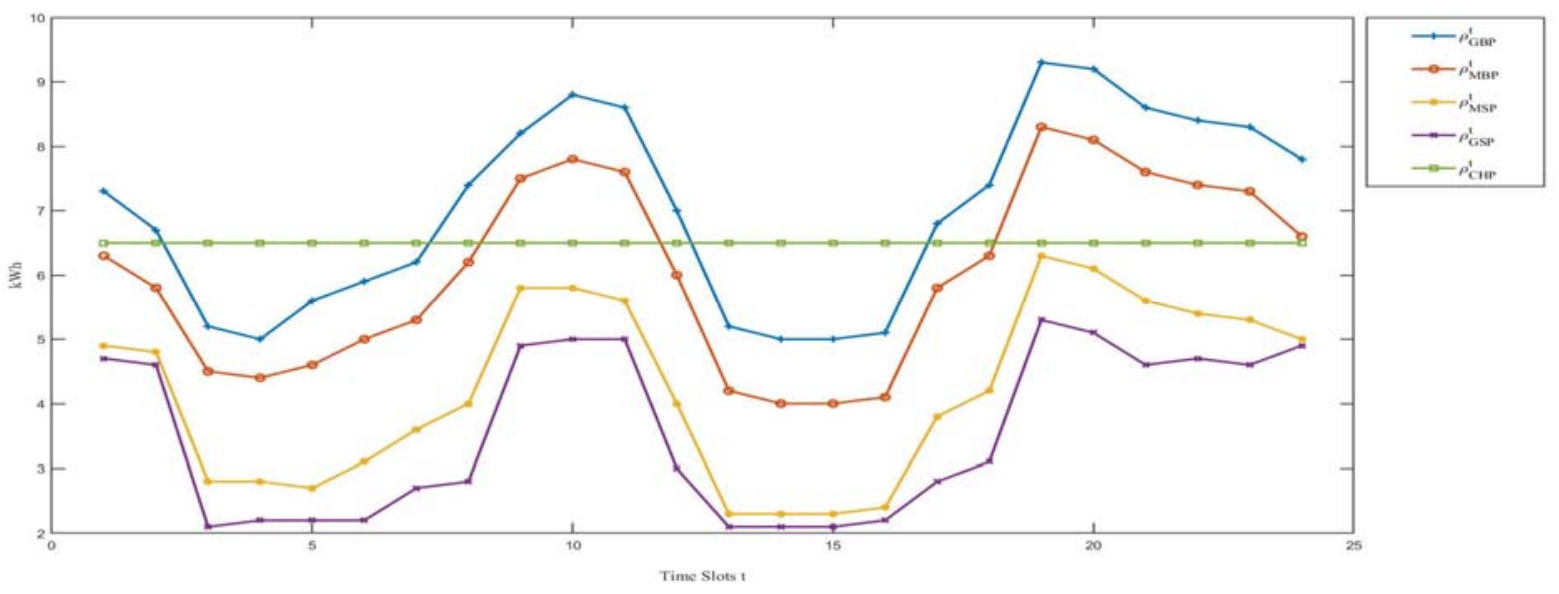

Fig. 3. Internal / External Energy Trading Prices 

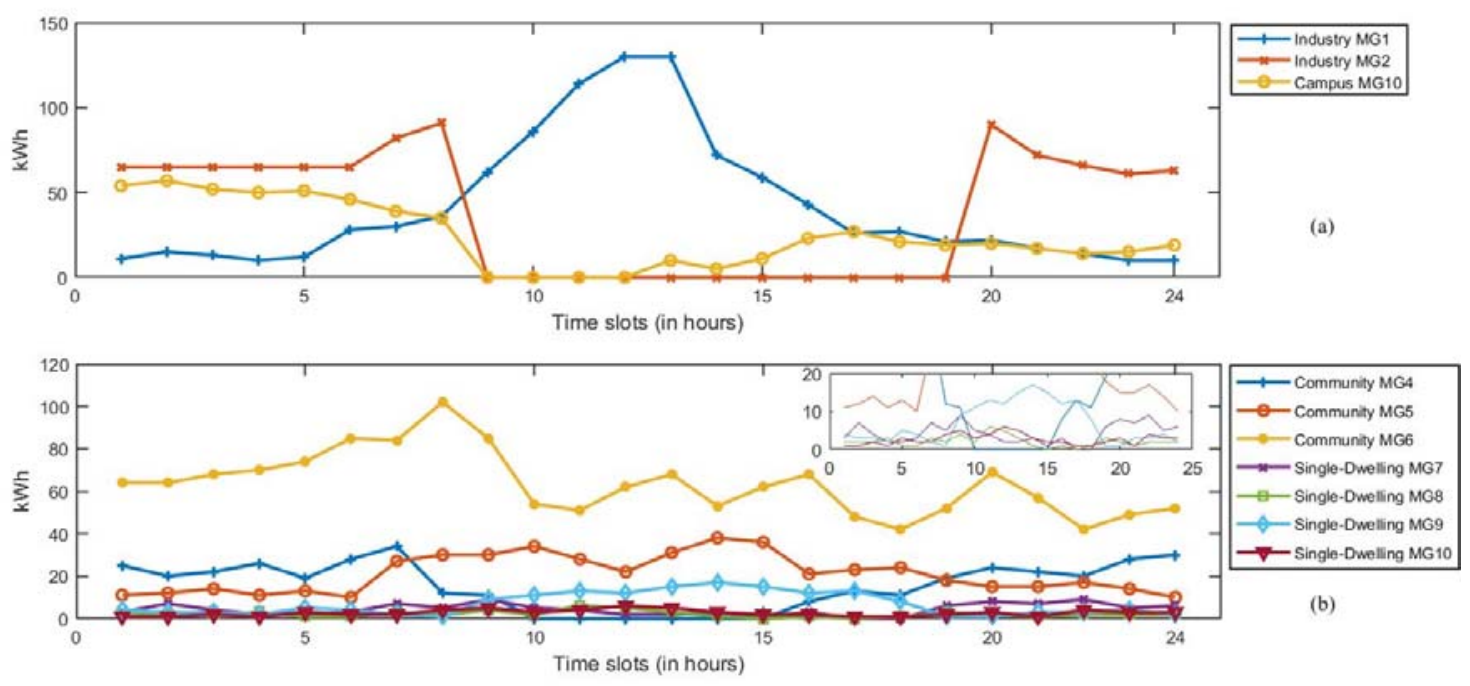

Fig. 4. (a) 24-hrs Load Profile of seller MG (b) 24-hrs Load Profile of buyer MG

\section{Results and discussion}

For the proposed work, a system with distributed smart microgrids is examined. We have made the following assumptions for simulation:

(1) There are $10 \mathrm{MGs}$ in the network out of which there are 2 industry MGs, 1 campus MG, 4 single-dwelling MGs and 3 community MGs (Table 1 ).

(2) Maximum and minimum microgrid buying prices $\left(\max \left(\rho^{M B P}\right)_{T} \& \min \left(\rho^{M B P}\right)_{T}\right)$ in a day are Rs. 8.5/Wh and Rs. 4/Wh, respectively.

(3) Energy production charges using CHP, $\rho_{C H P}^{t}=$ Rs. $6.5 / \mathrm{Wh}$

(4) Maximum and minimum CHP energy generation $\left(E_{C H P M A X}^{i, t} \& E_{C H P M I N}^{i, t}\right)$ is $200 \mathrm{kWh}$ and $80 \mathrm{kWh}$, respectively.

(5) The capacity of each ESS unit is expected to be $100 \mathrm{kWh}$.

(6) ESS's initial energy is considered to be $60 \mathrm{kWh}$.

(7) The capacity of EV battery is assumed to be $16 \mathrm{kWh}$.

(8) EV's initial battery energy is considered to be $8 \mathrm{kWh}$.

(9) ESS's/EV's charging and discharging efficiency $\left(\eta^{c} \& \eta^{d}\right)$ is set to 0.90.

(10) 24-hour day is split into 24 time slots.

The simulation is done using Python 3.6 on an Intel core i5 1.06 GHz CPU running Windows 10. Fig. 3. shows the internal and external energy trading prices in a day. Fig. 4. depicts the 24-hour load profile of seller and buyer microgrids in a typical working day.

\subsection{Results of Step 1 (Optimization):}

Table 2 shows the energy requirement (deficit) and excess energy (surplus) of each MG in the network obtained after each MG performs optimization as discussed in the above section. The energy requirement is indicated by negative value and excess energy is indicated by positive value. Since, CHP and PV energy generators are present in industry and campus microgrid, we have assumed that the energy requirement of these $\mathrm{MGs}_{(\mathrm{MG}}\left(\mathrm{MG}_{1}\right.$, $\mathrm{MG}_{2}$ and $\mathrm{MG}_{10}$ ) is fulfilled by the local energy generators. Therefore, $\mathrm{MG}_{1}, \mathrm{MG}_{2}$ and $\mathrm{MG}_{10}$ always acts as a seller in the energy market, if there is an excess energy generation. Each MG sends its energy requirement or excess energy to the DSO which schedules energy. The energy store in ESS/EV is only used if $P_{d i s}^{t}>0.5$ and the ESS/EV are only charged if $P_{c h}^{t}>0.5$. CHP energy generation also depends on the market prices. If CHP energy generation cost is more than the internal/external energy trading prices, then CHP energy generation is kept to minimum and energy is bought from the market.

\subsection{Results of Step 2 (CFES):}

DSO divides the total excess energy equally among all the buyer MGs. By this equal energy distribution, except $\mathrm{MG}_{3}, \mathrm{MG}_{4}, \mathrm{MG}_{5}$ and $\mathrm{MG}_{6}$, the energy requirement of all other MGs is fulfilled. Therefore, only $\mathrm{MG}_{3}$, 
$\mathrm{MG}_{4}, \mathrm{MG}_{5}$ and $\mathrm{MG}_{6}$ participate in the auction process of CFES. Table 3 shows the energy requirement of the MGs participating in auctioning. We can observe from Table 3 that the energy requirement of $\mathrm{MG}_{3}, \mathrm{MG}_{4}, \mathrm{MG}_{5}$ and $\mathrm{MG}_{6}$ is 0 during time slot 11,12 and 13. During these time slot the excess energy is sold to the utility grid.

The simulation results and discussions demonstrate the usefulness of the suggested strategy in terms of reducing costs in a hybrid multi-microgrid system when compared to individual microgrid external trading with the utility grid. The comparison of cost incurred to all the buyer microgrids using the proposed strategy is shown in Table 4.

\begin{tabular}{|c|c|c|c|c|c|}
\hline $\begin{array}{l}\text { Time } \\
\text { Slot }\end{array}$ & $\mathbf{M G}_{3}$ & $\mathbf{M G}_{4}$ & $\mathbf{M G}_{5}$ & $\mathbf{M G}_{6}$ & $\begin{array}{l}\text { Total Energy } \\
\text { Requirement }\end{array}$ \\
\hline 1 & 6.43 & 0.00 & 45.43 & 0.00 & 51.86 \\
\hline 2 & 0.43 & 0.00 & 44.43 & 0.00 & 44.86 \\
\hline 3 & 3.43 & 0.00 & 49.43 & 0.00 & 52.86 \\
\hline 4 & 8.14 & 0.00 & 52.14 & 0.00 & 60.29 \\
\hline 5 & 0.71 & 0.00 & 55.71 & 0.00 & 56.43 \\
\hline 6 & 8.14 & 0.00 & 65.14 & 0.00 & 73.29 \\
\hline 7 & 12.43 & 5.43 & 62.43 & 0.00 & 80.29 \\
\hline 8 & 0.00 & 6.86 & 78.86 & 0.00 & 85.71 \\
\hline 9 & 0.00 & 0.33 & 55.33 & 0.00 & 55.67 \\
\hline 10 & 0.00 & 0.00 & 0.50 & 0.00 & 0.50 \\
\hline 11 & 0.00 & 0.00 & 0.00 & 0.00 & 0.00 \\
\hline 12 & 0.00 & 0.00 & 0.00 & 0.00 & 0.00 \\
\hline 13 & 0.00 & 0.00 & 0.00 & 0.00 & 0.00 \\
\hline 14 & 0.00 & 0.00 & 2.50 & 0.00 & 2.50 \\
\hline 15 & 0.00 & 0.00 & 18.00 & 0.00 & 18.00 \\
\hline 16 & 0.00 & 0.00 & 40.00 & 0.00 & 40.00 \\
\hline 17 & 0.00 & 0.67 & 25.67 & 0.00 & 26.33 \\
\hline 18 & 0.00 & 4.67 & 22.67 & 0.00 & 27.33 \\
\hline 19 & 13.29 & 12.29 & 46.29 & 0.29 & 72.14 \\
\hline 20 & 5.14 & 0.00 & 50.14 & 0.00 & 55.29 \\
\hline 21 & 6.86 & 0.00 & 41.86 & 0.00 & 48.71 \\
\hline 22 & 6.57 & 3.57 & 28.57 & 0.00 & 38.71 \\
\hline 23 & 15.71 & 1.71 & 36.71 & 0.00 & 54.14 \\
\hline 24 & 16.86 & 0.00 & 38.86 & 0.00 & 55.71 \\
\hline
\end{tabular}

Table 3. Energy Requirement of MGs for auctioning during CFES

\begin{tabular}{|c|l|l|l|l|l|l|l|}
\hline Cost Incurred & $\mathbf{M G}_{\mathbf{3}}$ & $\mathbf{M G}_{\mathbf{4}}$ & $\mathbf{M G}_{\mathbf{5}}$ & $\mathbf{M G}_{\mathbf{6}}$ & $\mathbf{M G}_{7}$ & $\mathbf{M G}_{\mathbf{8}}$ & $\mathbf{M G}_{\mathbf{9}}$ \\
\hline $\begin{array}{c}\text { Without using } \\
\text { proposed method }\end{array}$ & 2654 & 3493.4 & 10522.2 & 562.4 & 202.2 & 264.6 & 236.7 \\
\hline $\begin{array}{c}\text { Using the proposed } \\
\text { method }\end{array}$ & 2443.07 & 3023.09 & 9370.42 & 489.39 & 175.6 & 228.5 & 204.5 \\
\hline $\begin{array}{c}\text { Cost Reduction } \\
\text { (Rs.) }\end{array}$ & 210.93 & 470.31 & 1151.78 & 73.01 & 26.6 & 36.1 & 32.2 \\
\hline
\end{tabular}

Table 4. Comparison of cost

\section{Conclusions}

This paper presents an energy scheduling strategy for a hybrid network of multi-microgrid. All the microgrids try to optimize the energy cost subject to CHP energy generation cost and ESS/EV charging/discharging cost. CHP energy generation depends on the energy market prices. If the energy buying price is higher than the CHP generation cost then the microgrid will prefer consuming CHP generated energy instead of buying energy from the market. Conversely, if the energy buying cost is less than CHP energy generation cost than the microgrid will let the CHP to generate maximum of its capacity and will sell the excess 
energy in the energy market. After optimization, the shortfall/excess energy data is sent to the DSO which schedules energy for each microgrid using CFES algorithm. CFES algorithm schedules energy fairly among all the buyer microgrids so that the cost of buying energy is greatly reduced. The simulation results show that the cost of buying energy is reduced greatly using the proposed strategy.

\section{Acknowledgements}

This work was supported by Visvesvaraya Ph.D. Scheme through the Ministry of Electronics and Information Technology (MeitY) by the Government of India under Grant MEITY-PHD-3097.

\section{References}

[1] A. Hirsch, Y. Parag, and J. Guerrero, "Microgrids: A review of technologies, key drivers, and outstanding issues," Renewable and Sustainable Energy Reviews, vol. 90, no. March, pp. 402-411, 2018, doi: 10.1016/j.rser.2018.03.040.

[2] F. Martin-Martínez, A. Sánchez-Miralles, and M. Rivier, "A literature review of Microgrids: A functional layer based classification," Renewable and Sustainable Energy Reviews, vol. 62, pp. 1133-1153, 2016, doi: 10.1016/j.rser.2016.05.025.

[3] A. H. Fathima and K. Palanisamy, "Optimization in microgrids with hybrid energy systems - A review," Renewable and Sustainable Energy Reviews, vol. 45, pp. 431-446, 2015, doi: 10.1016/j.rser.2015.01.059.

[4] M. F. Zia, E. Elbouchikhi, and M. Benbouzid, "Microgrids energy management systems: A critical review on methods, solutions, and prospects," Applied Energy, vol. 222. Elsevier Ltd, pp. 1033-1055, Jul. 15, 2018. doi: 10.1016/j.apenergy.2018.04.103.

[5] K. Techato and J. M. Guerrero, "Microgrid Transactive Energy : Review, Architectures, Distributed Ledger Technologies , and Market Analysis," pp. 1-23, 2020, doi: 10.1109/ACCESS.2020.2968402.

[6] O. Abrishambaf, F. Lezama, P. Faria, and Z. Vale, "Towards transactive energy systems: An analysis on current trends," Energy Strategy Reviews, vol. 26, p. 100418, 2019, doi: 10.1016/j.esr.2019.100418.

[7] B. Ramachandran, S. K. Srivastava, C. S. Edrington, and D. A. Cartes, "An intelligent auction scheme for smart grid market using a hybrid immune algorithm," IEEE Transactions on Industrial Electronics, vol. 58, no. 10, pp. 4603-4612, 2011, doi: 10.1109/TIE.2010.2102319.

[8] A. Loni and F. A. Parand, "A survey of game theory approach in smart grid with emphasis on cooperative games," 2017 IEEE International Conference on Smart Grid and Smart Cities, ICSGSC 2017, pp. 237-242, 2017, doi: 10.1109/ICSGSC.2017.8038583.

[9] W. Saad, Z. Han, H. V. Poor, and T. Başar, "Game-theoretic methods for the smart grid: An overview of microgrid systems, demandside management, and smart grid communications," IEEE Signal Processing Magazine, vol. 29, no. 5, pp. 86-105, 2012, doi: 10.1109/MSP.2012.2186410.

[10] W. Tushar et al., "Three-Party Energy Management with Distributed Energy Resources in Smart Grid," IEEE Transactions on Industrial Electronics, vol. 62, no. 4, pp. 2487-2498, 2015, doi: 10.1109/TIE.2014.2341556.

[11] W. Saad, Z. Han, T. Basar, Y. Wang, and H. V. Poor, "A Game-Theoretic Approach to Energy Trading in the Smart Grid," IEEE Transactions on Smart Grid, vol. 5, no. 3, pp. 1439-1450, 2014, doi: 10.1109/tsg.2013.2284664.

[12] N. Liu, X. Yu, C. Wang, and J. Wang, "Energy Sharing Management for Microgrids with PV Prosumers: A Stackelberg Game Approach," IEEE Transactions on Industrial Informatics, vol. 13, no. 3, pp. 1088-1098, 2017, doi: 10.1109/TII.2017.2654302.

[13] J. Lee, J. Guo, J. K. Choi, and M. Zukerman, "Distributed energy trading in microgrids: A game-theoretic model and its equilibrium analysis," IEEE Transactions on Industrial Electronics, vol. 62, no. 6, pp. 3524-3533, 2015, doi: 10.1109/TIE.2014.2387340.

[14] S. Park, J. Lee, S. Bae, G. Hwang, and J. K. Choi, "Contribution-Based Energy-Trading Mechanism in Microgrids for Future Smart Grid: A Game Theoretic Approach,” IEEE Transactions on Industrial Electronics, vol. 63, no. 7, pp. 4255-4265, 2016, doi: 10.1109/TIE.2016.2532842.

[15] A. M. Jadhav and N. R. Patne, "Priority-Based Energy Scheduling in a Smart Distributed Network with Multiple Microgrids," IEEE Transactions on Industrial Informatics, vol. 13, no. 6, pp. 3134-3143, 2017, doi: 10.1109/TII.2017.2671923.

[16] N. A. Funde, M. M. Dhabu, P. S. Deshpande, and N. R. Patne, "SF-OEAP: Starvation-free optimal energy allocation policy in a smart distributed multimicrogrid system," IEEE Transactions on Industrial Informatics, vol. 14, no. 11, pp. 4873-4883, 2018, doi: 10.1109/TII.2018.2810816

[17] H. S. V. S. K. Nunna and D. Srinivasan, "Multiagent-Based Transactive Energy Framework for Distribution Systems with Smart Microgrids," IEEE Transactions on Industrial Informatics, vol. 13, no. 5, pp. 2241-2250, 2017, doi: 10.1109/TII.2017.2679808.

[18] N. Funde, M. Dhabu, and P. Deshpande, "CLOES: cross-layer optimal energy scheduling mechanism in a smart distributed multimicrogrid system," Journal of Ambient Intelligence and Humanized Computing, vol. 11, no. 11, pp. 4765-4783, 2020, doi: 10.1007/s 12652-020-01745-1.

[19] K. Jain, M. Dhabu, O. Kakde, and N. Funde, "Completely fair energy scheduling mechanism in a smart distributed multi-microgrid system," Journal of King Saud University - Computer and Information Sciences, Aug. 2021, doi: 10.1016/J.JKSUCI.2021.08.002.

Authors Profile

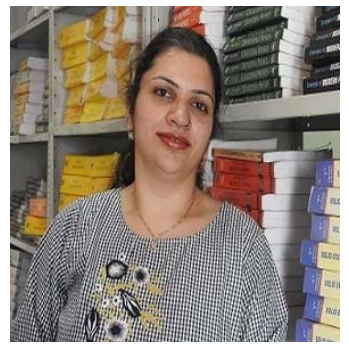

Khushboo Jain is pursuing her Ph.D. from Computer Science and Engineering Department, Indian Institute of Information Technology (IIIT), Nagpur, India. She is also currently working as an Adjunct Assistant Professor at IIIT, Nagpur, India. She has more than 10 years of teaching experience. She received her B. E. and M. Tech. degree from Nagpur University, Nagpur, India in 2008 and 2011 respectively. Her areas of interest include Smart Grids, Energy Management, Natural Language Processing and Machine Learning. She is a member of ISTE Society. 


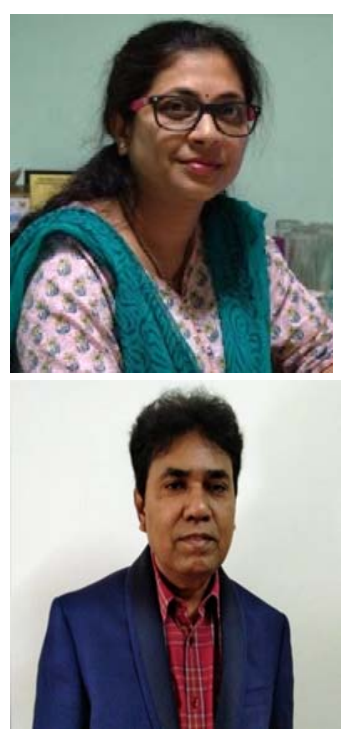

Meera M. Dhabu received Ph.D. from Visvesvaraya National Institute of Technology, Nagpur, India. She is currently an Assistant professor with the Department of Computer Science and Engineering at Visvesvaraya National Institute of Technology, Nagpur, India. Her research interests include Data mining, Machine learning and Smart Grid. She has 18 years of academic experience. She has co-authored a number of research articles in various journals, conferences, and book chapters. She is a member of IEEE and ACM.

Omprakash Kakde received Ph.D. from Visvesvaraya National Institute of Technology, Nagpur, India. He is currently Director of Indian Institute of Information Technology (IIIT), Nagpur, India. Prior to this, he was the Director of Veermata Jijabai Technological Institute (VJTI), Mumbai, India. He has more than 25 years of academic experience. He has co-authored a number of research articles in various journals, conferences, and book chapters. His area of research includes Language Processor, Smart Grids and Machine Learning. 